\title{
LNA probes substantially improve the detection of bacterial endosymbionts in whole mount of insects by fluorescent in-situ hybridization
}

\author{
Natarajan Gayatri Priya, Neeti Pandey and Raman Rajagopal*
}

\begin{abstract}
Background: Detection of unculturable bacteria and their localization in the host, by fluorescent in-situ hybridization (FISH), is a powerful technique in the study of host-bacteria interaction. FISH probes are designed to target the $16 \mathrm{~s}$ rRNA region of the bacteria to be detected. LNA probes have recently been used in FISH studies and proven to be more efficient. To date no report has employed LNA probes for FISH detection of bacterial endosymbiont in the whole mount tissues. Further, though speculated, bacteriocytes have not been reported from males of Bemisia tabaci.
\end{abstract}

Results: In this study, we compared the efficiency in detecting bacteria by fluorescent DNA oligonucleotides versus modified probes containing Locked Nucleic Acid (LNA) substitution in their structure. We used the insect Bemisia tabaci as the experimental material since it carried simultaneous infection by two bacteria: one a primary endosymbiont, Portiera (and present in more numbers) while the other a secondary endosymbiont Arsenophonus (and present in less numbers). Thus a variation in the abundance of bacteria was expected. While detecting both the bacteria, we found a significant increase in the signal whenever LNA probes were used. However, the difference was more pronounced in detecting the secondary endosymbiont, wherein DNA probes gave weak signals when compared to LNA probes. Also, signal to noise ratio for LNA probes was higher than DNA probes. We found that LNA considerably improved sensitivity of FISH, as compared to the commonly used DNA oligonucleotide probe.

Conclusion: By employing LNA probes we could detect endosymbiotic bacteria in males, which have never been reported previously. We were able to detect bacteriocytes containing Portiera and Arsenophonus in the males of B. tabaci. Thus, employing LNA probes at optimized conditions will help to significantly improve detection of bacteria at the lowest concentration and may give a comprehensible depiction about their specific distribution within samples.

\section{Background}

Microscopic detection and localization of a specific DNA or RNA segment within single cells or a histological section has been made possible with the advent of the In-Situ Hybridization (ISH) technique. This technique relies principally on formation of Watson-Crick base pairing between the gene of interest and the applied complementary sequence to which the reporter molecule is attached [1]. Fluorescent in-situ hybridization (FISH)

\footnotetext{
* Correspondence: zoorajagopal@gmail.com

Gut Biology Lab, Department of Zoology, University of Delhi, Room No 117, Delhi 110007, India
}

\section{Biomed Central}

(c) 2012 Priya et al.; licensee BioMed Central Ltd. This is an Open Access article distributed under the terms of the Creative Commons Attribution License (http://creativecommons.org/licenses/by/2.0), which permits unrestricted use, distribution, and reproduction in any medium, provided the original work is properly cited. is an extension of this technique in which a fluorophore tagged to the probe, acts as the reporter molecule. FISH is a widely used technique in clinical studies relating to diagnosis, prognosis and sometimes, even remission of diseases like cancer [2,3]. In microbiology, studies pertaining to microbial ecology employ FISH in detection and identification of unculturable microbes in clinical and environmental samples as well as whole mount tissues $[4,5]$. Some studies have employed FISH for revealing the distribution pattern of two very closely related ( $<3 \%$ difference in nucleotide sequence) species of marine cyanobacteria [6]. DNA oligonucleotide probes are most commonly used when compared to ssDNA, dsDNA or 
RNA probes due to features like: stability, ease of availability and cost effectiveness.

A modification in the structure of nucleotides with methylene bridge to connect 2' oxygen and 4' carbon of the ribose ring, gives rise to Locked Nucleic Acid (LNA) [7]. The extra bridge in an LNA structure makes the ribose moiety inaccessible, thereby locking the structure to high binding affinity conformation $[8,9]$. Such LNA nucleotides can be mixed with DNA or RNA residues during synthesis of oligonucleotide to enhance the hybridization specificity, sensitivity and duplex stability $[8,10]$. When compared to DNA only oligonucleotide probes, it is seen that LNA modified DNA oligonucleotide probes (hereinafter called LNA probes) are 10 fold more sensitive when applied in techniques like northern analysis [11]. LNA probes have also been successfully used for FISH to identify individual E. coli cells [12]. They have been used for temporal and spatial detection of miRNAs or mRNA by whole mount ISH and in tissue sections [13-16]. Some studies have used LNA in clinical studies for detection and differentiation between two fungal pathogens in tissue sections [17-19]. There are many reports that have identified and localized bacteria by targeting $16 \mathrm{~S}$ rRNA gene in whole mount or microtome section samples but till date there has been no report wherein LNA probes have been employed for bacterial detection by FISH in whole mount or microtome section of biological samples.

The insect Bemisia tabaci, commonly known as whitefly, is an agricultural pest with a wide host range. $B$. tabaci is a vector of a group of plant viruses known as Geminiviruses which significantly damage the host plant. Recent studies have linked the transmission of Tomato Yellow Leaf Curl virus (TYLCV), to the GroEL protein of a secondary endosymbiont of B. tabaci [20]. Therefore, an extensive study of the type and nature of spread of $B$. tabaci endosymbionts is primary to understanding their functional role within the host insect. Two types of endosymbionts are reported to be present within the $B$. tabaci, namely the primary endosymbiont and the secondary endosymbiont [21]. Whiteflies are one of the rare cases in which co-infection, of primary and secondary symbionts, occurs in the same cell [22]. Therefore, in this study we have compared the efficiency of both DNA only and LNA modified DNA probes in the detection and localization of a primary endosymbiont that is present in abundance, as well as a secondary endosymbiont that is less abundant in nature.

\section{Methods}

We collected adult Bemisia tabaci from cotton leaves from fields of Indian Agricultural Research Institute (Pusa, New Delhi, India), washed them with ethanol and water, and stored in acetone at $-20^{\circ} \mathrm{C}$ till further processing. The specimens were processed using standardized method of Gottlieb et al [21] for whitefly with slight modifications. $B$. tabaci specimens were stored overnight in Carnoy's fixative (chloroform: ethanol: glacial acetic acid, 6:3:1) and decolorized with $6 \% \mathrm{H}_{2} \mathrm{O}_{2}$ in ethanol for 24 hrs. Portiera and Arsenophonus detection was performed using FAM labeled probe bearing 5' TGTCAGTGTCAGCCCAG AAG 3' sequence and TYE-665 probe bearing of 5' TCAT GACCACAACCTCCAAA 3' sequence respectively [20]. The DNA probe and modified LNA were supplied by Exiqon A/S [the exact positions of the LNA modifications of Portiera (batch no. 5032716, containing 5 LNA) and Arsenophonus (batch no. 503274, containing 6 LNA), are not known to us]. The decolorized insects were hybridized at $40^{\circ} \mathrm{C}$, with the DNA and LNA probes, in hybridization buffer (20 mM Tris-Cl [pH 8.0], $0.9 \mathrm{M} \mathrm{NaCl}, 0.01 \%$ sodium dodecyl sulfate) containing increasing amount of formamide (0\%-80\%). Probe concentrations of 0.6 pmoles for Portiera and 1.0 pmoles for Arsenophonus were kept identical for LNA and DNA. After the overnight incubation, the samples were thoroughly washed in a washing buffer $(0.3 \mathrm{M} \mathrm{NaCl}, 0.03 \mathrm{M}$ sodium citrate, $0.01 \%$ sodium dodecyl sulfate) for 5 minutes and mounted using Vectashield (Vector Labs). Each of the endosymbiont was detected at 9 different formamide concentrations $(0 \%$ $80 \%)$ separately, with DNA as well as LNA probes. Replicates consisted of 10 insects for each condition. Specificity of detection was confirmed using no probe staining and RNase- digested specimen staining. All the images were acquired at fixed camera and microscope settings for DNA and LNA with Nikon A1 confocal microscope. The fluorescence intensities were quantified by NIS elements (V 3.21.02) image analysis software (Nikon).

\section{Results and discussion}

The primary endosymbiont of Bemisia tabaci is Portiera [23]. This symbiont is housed exclusively in specialized structures called bacteriocytes [24]. Since this insect cannot survive without its obligate primary endosymbiont, these symbionts are present in higher proportion or abundance than other secondary endosymbionts. FISH studies pertaining to localization of Portiera using confocal microscope has been described earlier [21]. Arsenophonus is a secondary endosymbiont whose exact role is yet to be ascertained and whose population within the insect is lower than that of Portiera. Location of Arsenophonus is reported to be in the same cell as Portiera i.e. the bacteriocytes [22].

\section{Comparing LNA and DNA probes to detect Portiera the primary bacterial endosymbiont of Bemisia tabaci}

While detecting Portiera we found LNA to be more sensitive than DNA oligonucleotide probes (Figure 1). At $0 \%$ formamide concentration, we observed very high 

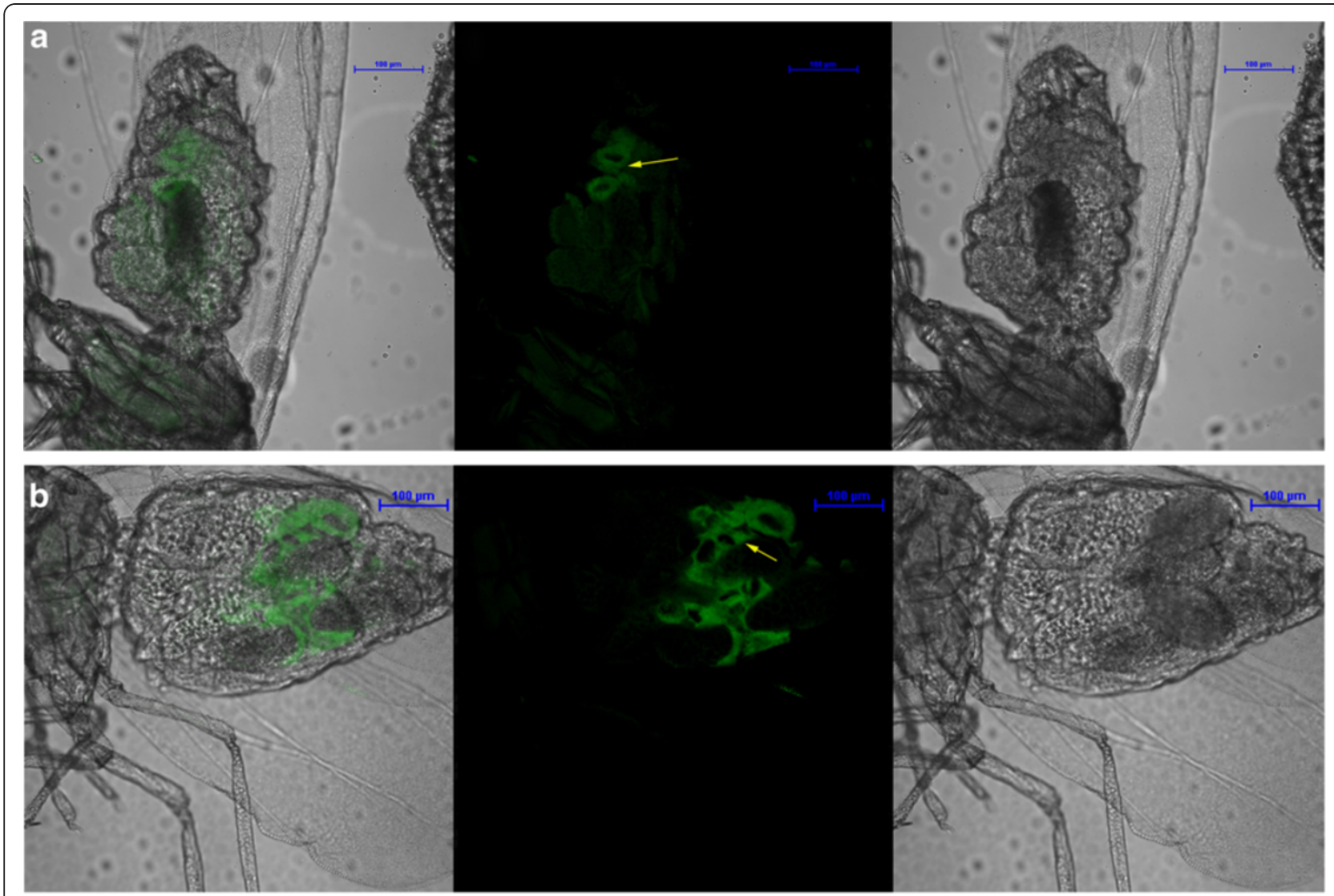

Figure 1 FISH staining of Portiera 16 S rRNA in whole mount of whitefly Bemisia tabaci. FAM labeled oligonucleotide DNA probe and modified LNA probes were used to detect Portiera in B. tabaci. (A.b) DNA probe stains for Portiera in the bacteriocytes (B.b) at the same concentration (0.6 pmoles) LNA probe shows higher signal and lower background while staining for Portiera. Arrows indicate the bacteriocytes. The images have been taken at best formamide concentration for Portiera DNA (40\%) and LNA (60\%) probes separately. Both DNA and LNA panels also show merged and DIC images (as a and c respectively). All the images were acquired at fixed camera and microscope settings with Nikon A1 confocal microscope.

DNA and LNA signals, but these samples also showed very high background noise [12] and hence we excluded it from analysis. DNA probe had highest intensity values $(\sim 30,000)$ at $30 \%$ formamide concentration (Figure 2$)$. All intensity measurements were done after background correction. Previous studies [25] with DNA probes detecting Portiera have used $30 \%$ formamide concentration for their FISH experiments, which is in agreement to our result obtained from DNA probe. The LNA signals $(\sim 70,000)$ peaked at $50 \%$ formamide concentration. The signal intensities of both DNA and LNA probes varied only to some extent with increasing formamide concentrations. Negative controls did not show any signal for Portiera (Additional file 1: Figure S1 \& Additional file 2: Figure S2). Overall, it was clearly evident that in most of the formamide concentrations, LNA probes had signal intensity nearly 2 times (and sometimes even more) as high as its DNA counterpart when detecting Portiera.
The Signal to Noise $(\mathrm{S} / \mathrm{N})$ value is an indicator of sensitivity of the probe since it is a measure of both the signal and the background. For this purpose no background correction was done, so that along with the actual signals of Portiera, the background noise of DNA and LNA could also be calculated for the same samples for $100 \mu^{2}$ area respectively. $\mathrm{S} / \mathrm{N}$ ratio value was obtained by dividing signal intensity with the background noise. Figure 3 , where $\mathrm{S} / \mathrm{N}$ ratio is plotted against increasing formamide concentration compares the two probes. The LNA probe had nearly twice as much $\mathrm{S} / \mathrm{N}$ values as DNA probe, while detecting Portiera. The highest $\mathrm{S} / \mathrm{N}$ value (823) was obtained with LNA probe at $60 \%$ formamide concentration. Use of high formamide concentration for LNA probes in order to reduce the background noise, has been previously performed when detecting lactic acid bacteria [26]. In DNA probe the highest $\mathrm{S} / \mathrm{N}$ value (334) was at $40 \%$ formamide concentration. It was evident from the graph 


\section{Portiera detection by LNA and DNA probes}

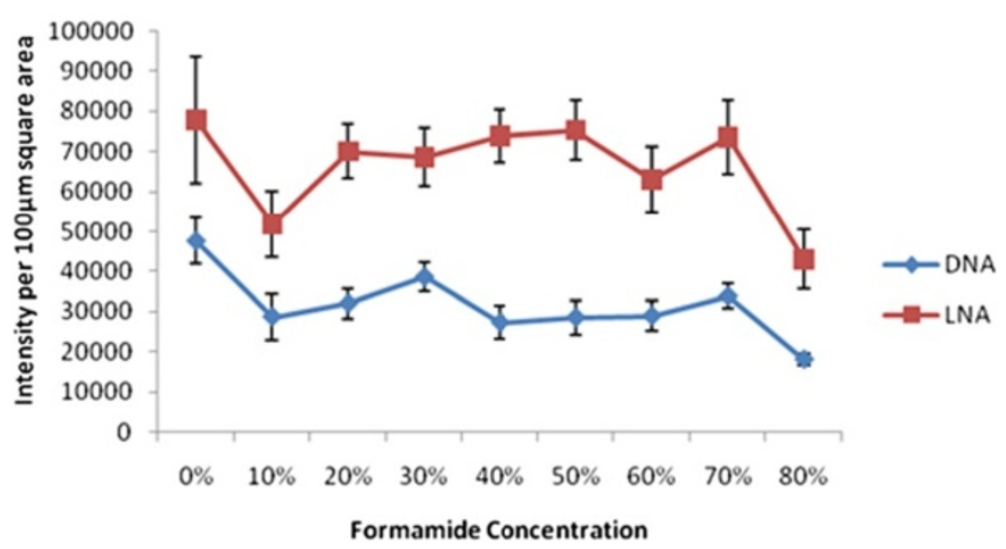

Figure 2 Comparison between LNA and DNA probes while detecting the more abundant endosymbiont (Portiera). This graph depicts signal intensity profiles of LNA and DNA probes as a function of formamide concentration after background subtraction. At the same concentration, the intensity profile of LNA probe is significantly higher than the DNA probe while detecting Portiera, an endosymbiont of high abundance. All the images were acquired at fixed camera and microscope settings for DNA and LNA with Nikon A1 confocal microscope. Fluorescence intensities were quantified by NIS elements (V 3.21.02) image analysis software (Nikon).

that the LNA probe has higher signal and lower noise ratio than DNA at all formamide concentrations. At $0 \%$ formamide concentration even though the main signal is high, an equally high background noise reduces the $\mathrm{S} / \mathrm{N}$ ratio value in both DNA and LNA probes. In agreement to previous studies [12], we find that high sensitivity and stringency can be obtained by using LNA probes at high formamide concentrations while performing FISH in insect whole mounts.

Comparing LNA and DNA probes to detect Arsenophonus the secondary bacterial endosymbiont of Bemisia tabaci FISH detection of Arsneophonus $16 \mathrm{~S}$ rRNA was performed keeping all the conditions, but the laser settings,

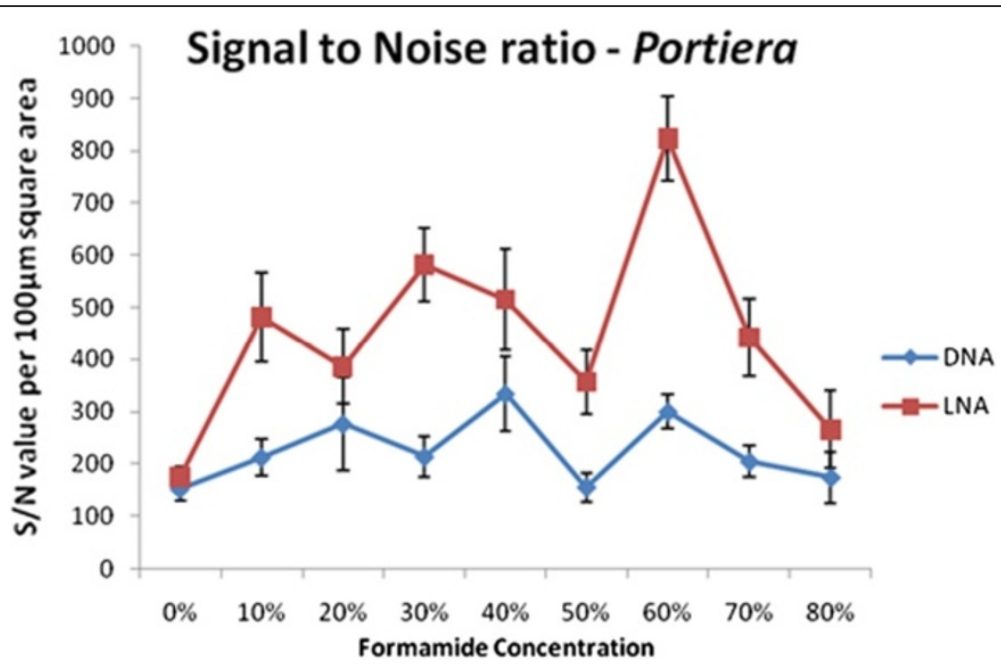

Figure 3 Signal to noise ratio of LNA and DNA probes while detecting the more abundant endosymbiont (Portiera). This graph depicts the signal to noise ratio, per $100 \mu \mathrm{m}$ square area and plotted against increasing formamide concentration. No background correction was performed here. The value was calculated by dividing signal with the background of the same image and thus it gives a good idea about the binding efficiency of the probe. Here, LNA probe has a high signal to noise ratio at $60 \%$ formamide concentration followed by $30 \%$ formamide concentration, when compared to DNA probe. The signal of LNA probe is always high than the DNA probe at all formamide concentrations. Portiera was detected at 9 different formamide concentrations (0\%-80\%), both by DNA as well as the LNA probes. Fluorescence intensities were quantified by NIS elements (V 3.21.02) image analysis software (Nikon). 
similar for DNA and LNA probes (Figure 4). This was done because, at laser settings where we could pick up signals of LNA, DNA did not show any detection signal (Additional file 3: Figure S3A and S3B). Also, when the laser power, $\mathrm{HV}$ and offset were increased with regard to DNA probe, LNA probe increased multifold in signal intensity and background (Additional file 3: Figure S3C). The laser settings were then lowered for LNA probe to such an extent that even the lowest signal produced by LNA was detectable. Different probe concentrations were also tested for DNA and LNA in order for detecting Arsenophonus where 1 pmoles concentration showed good results. At lower probe concentration (0.6pmoles) that was used for detection of Portiera, DNA failed to produce any signal for Arsenophonus, even though non-specific background signals could still be detected (Additional file 4: Figure S4A). LNA probe produced low intensity signals at the same concentration (Additional file 4: Figure S4B).

We found that LNA probes produced very high signals when compared to the DNA probes (Figure 4) while detecting Arsenophonus. We performed all the intensity measurements only after background correction. The LNA probe had highest intensity values $(>60,000)$ at $70 \%$ formamide concentration while the lowest $(30,000)$ at $10 \%$. DNA probe had highest intensity at $30 \%$ formamide concentration $(39,000)$ and lowest at $(16,000) 80 \%$ formamide concentration. At $10 \%$ formamide concentration, LNA signal was nearly as low as the DNA signal (Figure 5). The DNA probe gave an intensity which was similar to that of LNA probe at $0 \%$ formamide concentration. Similar to the earlier case of Portiera, $0 \%$ formamide gave high signal intensity as well as very high background noise. Therefore we did not consider it as an ideal concentration to detect the difference between the probes. It was seen that DNA probe produced good signal only at very low formamide concentration unlike LNA probe. Negative controls did not show any signal for Arsenophonus (Additional file 1: Figure S1 \& Additional file 2: Figure S2). Since high formamide concentration produces high stringency, false positive signals get negated while using LNA probes.
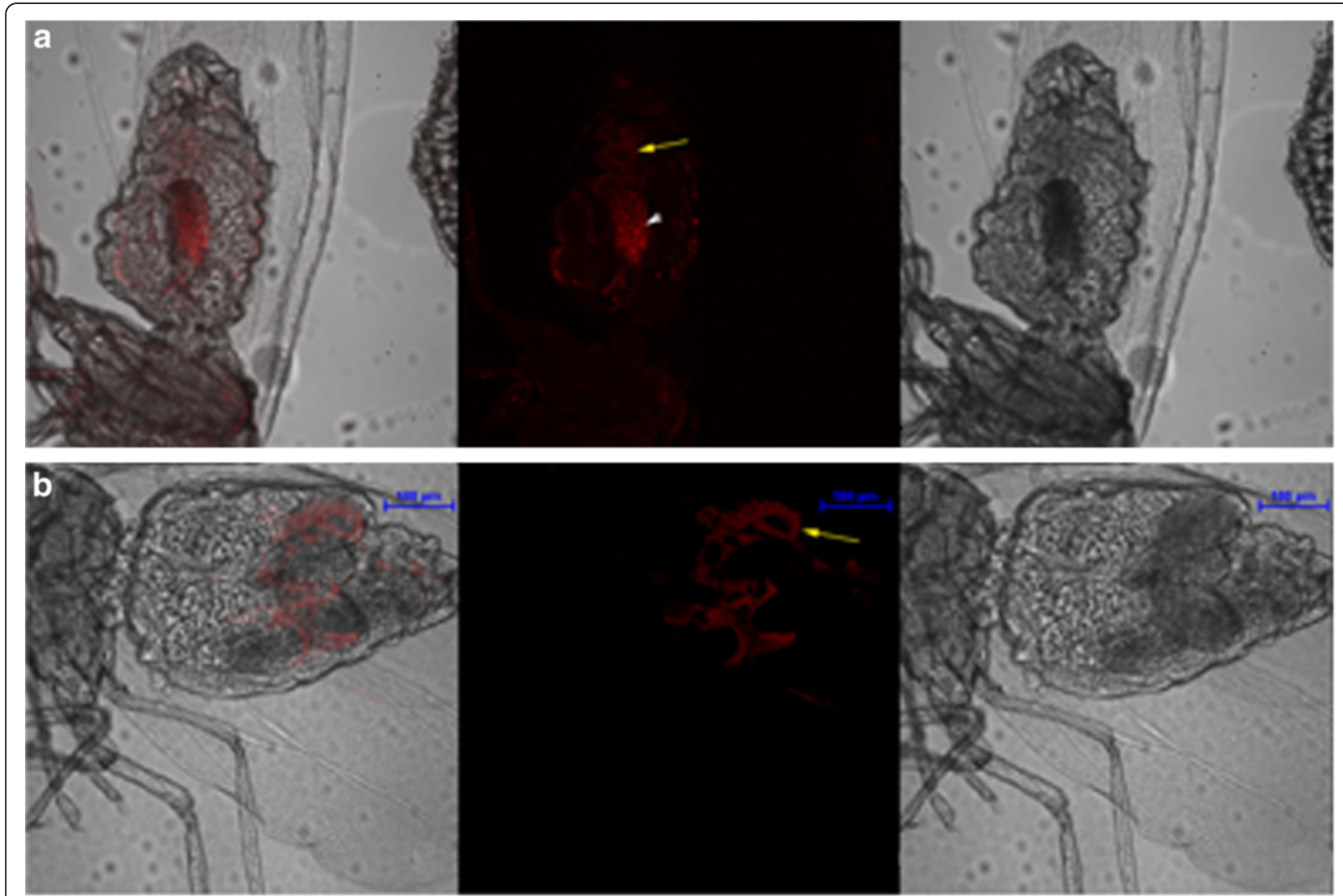

Figure 4 FISH staining of Arsenophonus 16 S rRNA in whole mount of whitefly Bemisia tabaci. (A.b) DNA probe stains Arsenophonus in the bacteriocytes; (B.b) at the same concentration (1.0 pmoles) LNA probe shows higher signal and a low background while staining for Arsenophonus. Arrows indicate the bacteriocytes. White arrowhead indicates the non-specific background in DNA samples. The images have been taken at best formamide concentration for Arsenophonus DNA (30\%) and LNA (70\%) probes separately. Both DNA and LNA panels also show merged and DIC images (as a and $\mathbf{c}$ respectively). 


\section{Arsenophonus detection by LNA and DNA probes}

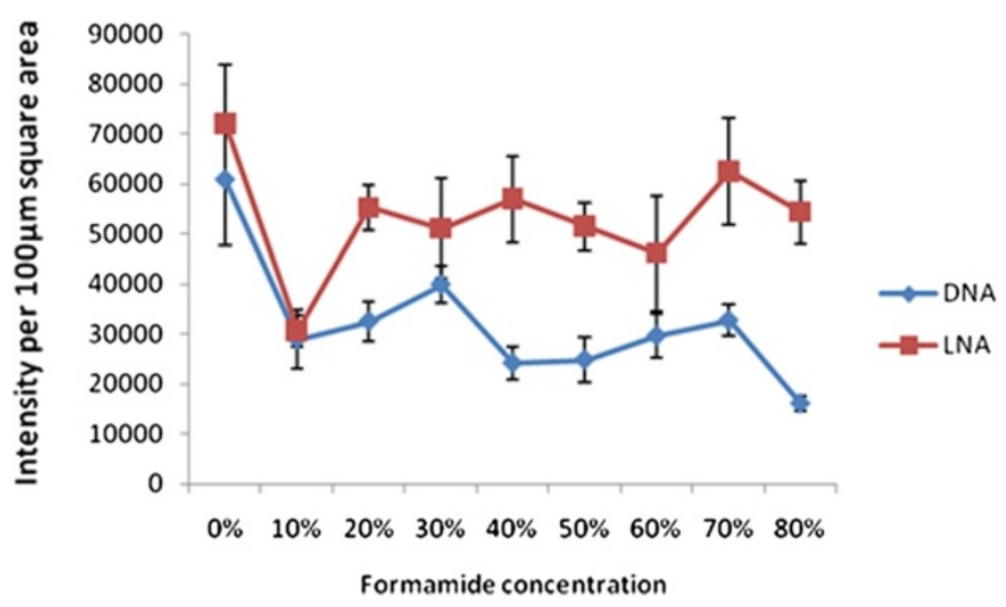

Figure 5 Comparison between LNA and DNA probes for detecting endosymbiont of lower abundance (Arsenophonus). All specimens were processed using the procedure described for Portiera. However, the probe concentration used for Arsenophonus was 1.0 pmoles and kept identical for LNA and DNA. This graph depicts the signal intensity profiles of LNA and DNA probes as a function of formamide concentration after background subtraction. At the same concentration, the intensity profile of LNA probe is significantly higher than the DNA probe while detecting Arsenophonus, an endosymbiont of low abundance. Fluorescence intensities were quantified by NIS elements (V 3.21.02) image analysis software (Nikon).

We then compared the sensitivity profiles of both the probes based on Signal to Noise $(\mathrm{S} / \mathrm{N})$ ratio. For $\mathrm{S} / \mathrm{N}$ ratio calculation, no background correction was performed, so that the background noise and actual signals could be recorded per $100 \mu \mathrm{m}^{2}$ area for both DNA and LNA probes in Arsenophonus samples. We calculated the $\mathrm{S} / \mathrm{N}$ ratio and found that LNA values were significantly higher than the DNA values (Figure 6). At $80 \%$ formamide concentration, the highest $\mathrm{S} / \mathrm{N}$ value of LNA probe (6852) was 20 times the $\mathrm{S} / \mathrm{N}$ values of DNA probe (331) at the same concentration. $60 \%$ formamide concentration was equally effective for LNA probes. The $\mathrm{S} / \mathrm{N}$ ratio value for LNA probe (602) dipped lower at $40 \%$ formamide concentration, which was still more

\section{Signal to Noise ratio- Arsenophonus}

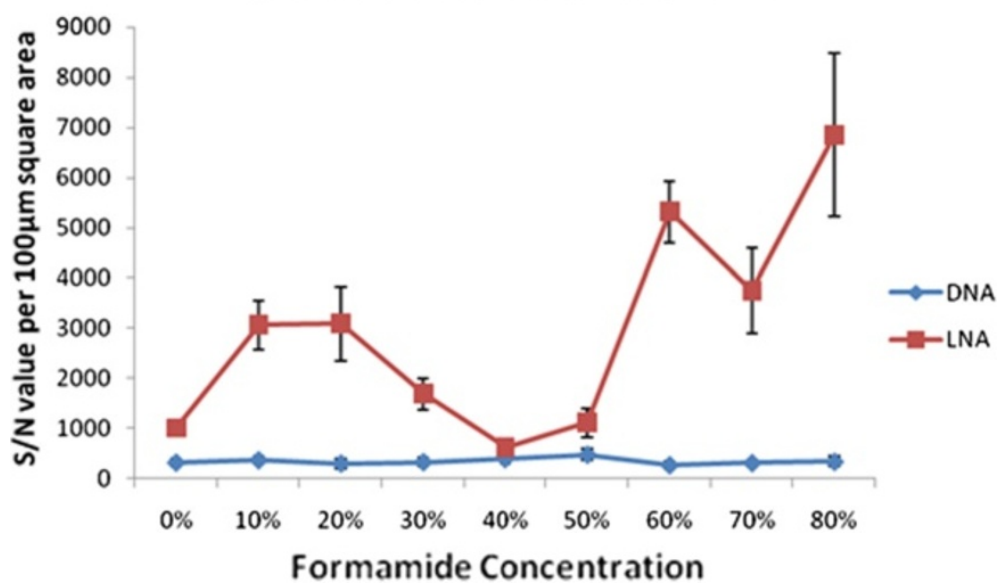

Figure 6 Signal to noise ratio of LNA and DNA probes while detecting the less abundant endosymbiont (Arsenophonus). The graph depicts the signal to noise ratio, per $100 \mu \mathrm{m}$ square area and plotted against increasing formamide concentration. No background correction was performed here. $\mathrm{S} / \mathrm{N}$ value was calculated by dividing signal with the background of the same image and thus it gives a good idea about the binding efficiency of the probe. LNA has a high signal to noise ratio at all formamide concentrations, when compared to DNA probe. The high signal and low background of LNA probes was observed even when the laser settings were lower than that of DNA probes. Arsenophonus was detected at 9 different formamide concentrations (0\%-80\%), both by DNA as well as the LNA probes. Replicates consisted of 10 insect samples for each condition. Fluorescence intensities were quantified by NIS elements (V 3.21.02) image analysis software (Nikon). 
than the S/N value of DNA probe (381) at the same formamide concentration. The DNA probe had highest S/N value (472) at $50 \%$ formamide concentration and lowest value (265) at $60 \%$ formamide concentration. It needs to be noted that the statistically important difference between LNA probe and DNA probe prevailed in spite of the low laser settings for former's detection. LNA probe detected Arsenophonus as sensitively as Portiera, irrespective of the endosymbiont's abundance, thereby proving its high efficiency compared to DNA probe.

The results presented here show that apart from many other applications reported so far [11-19], modified LNA probes are more effective for detecting bacteria in whole mounts of insect tissue than the conventional DNA oligonucleotide probes. This is because LNA probes are stable against 3'-exonucleolytic degradation and possess excellent aqueous solubility [27]. Additionally, the charged phosphate backbone of LNA oligonucleotides allows them to be transfected and taken up by cells just like DNA, thus finding use in many biological applications $[7,15,26]$. LNA modification of oligonucleotides reduces flexibility and results in more stable duplex structures [8]. The integration of 2-4 LNAs with oligonucleotides increases their binding to $16 \mathrm{~S}$ ribosomal RNA by up to 22-fold [12]. The improvement in detecting the endosymbionts of interest by LNA probes, when compared to DNA counterpart, is due to their increased thermodynamic stability and improved discrimination between perfectly matched and mismatched target nucleic acids [27]. It can be suggested that the features like higher melting temperature, better tissue penetrability and target accessibility [28] are the reasons why LNA outperforms DNA at nearly all formamide concentrations.

\section{Detection of bacteriocytes in male $B$. Tabaci}

Having concluded that LNA probes are better, we then tried to unravel more information than already reported regarding the distribution of endosymbionts using these probes. It has been reported that in B. tabaci, Portiera is present exclusively in the bacteriocytes and more so, easily detectable only in adult females [21]. Even though males are considered evolutionarily dead, due to the fact that they do not transmit symbionts to the offspring, studies in other insects like carpenter ants indicate that males do inherit endosymbionts for survival during their lifetime [29]. Earlier reports about bacterial symbiont localization have never reported any localization within males of B. tabaci [22,25]. Since from our previous results, $60 \%$ formamide concentration for both Portiera

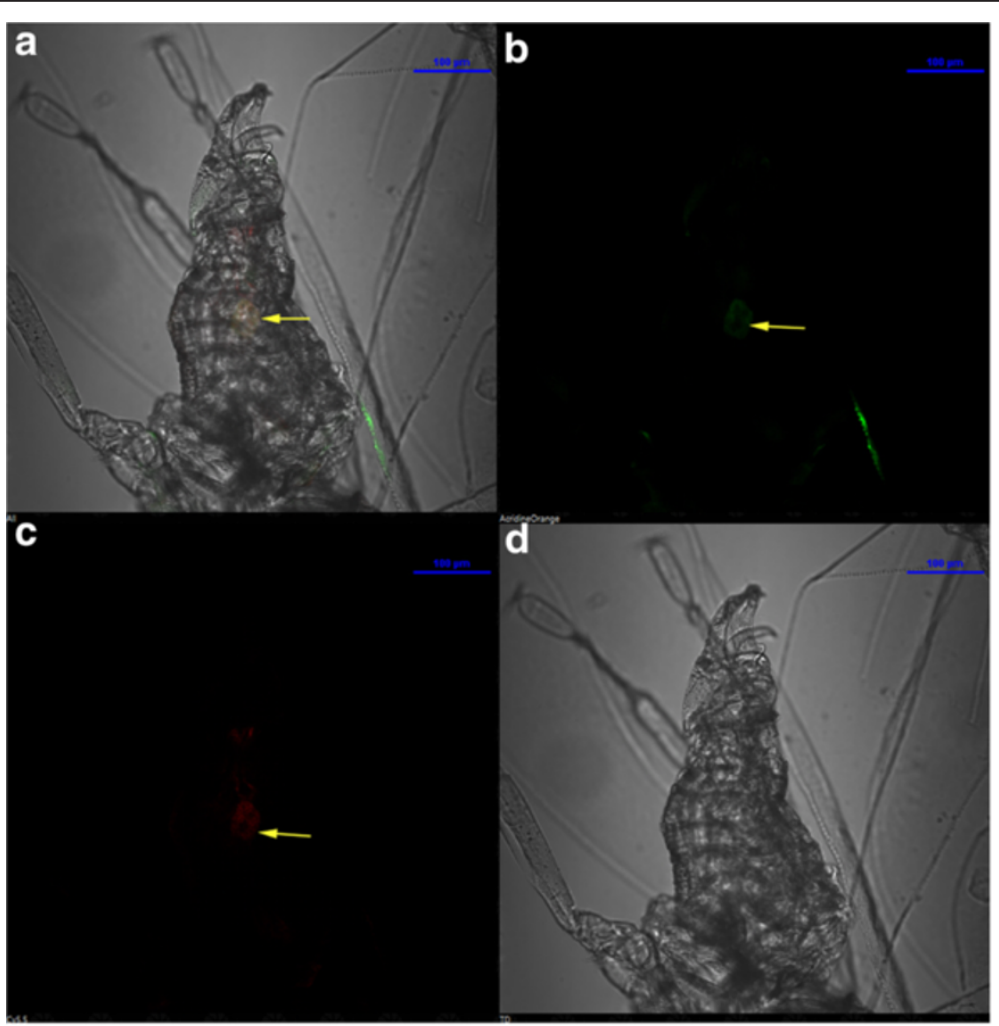

Figure 7 FISH staining of bacteriocyte in Bemisia tabaci male. The LNA probe details remain similar to those described in Figure 1 and 4. (A.b \& A.c) LNA probe stains Portiera and Arsenophonus in the bacteriocytes of adult male; Arrows in yellow indicate the bacteriocytes. The panel also shows merged and DIC images (as $\mathbf{A}$.a and $\mathbf{A}$.d respectively). 
and Arsenophonus produced high signal and low background, we considered it optimum for our investigation with LNA probes. We have detected for the first time, using LNA probes, not only Portiera but Arsenophonus signals as well, within the bacteriocytes of adult males (Figure 7). These endosymbionts, however, could not be detected when we used DNA oligonucleotide probes for staining.

\section{Conclusion}

Further studies using LNA probes for whole mount FISH can give us a better idea about the spread of endosymbionts and the various niches occupied by them within a tissue sample. In B. tabaci the use of LNA probes for detection of other endosymbionts will provide better understanding about the fly. Use of LNA can also be extended to the level of visualizing the existing interaction between the virus and the endosymbionts.

\section{Additional files}

Additional file 1: Figure S1. FISH staining of Portiera and Arsenophonus in whole mount of whitefly B. tabaci in RNase digested insect sample. No signal is detected for either Portiera (A.b) or Arsenophonus (A.C) when using LNA probes at similar conditions as in Figures 1 and 4 . $a$ and $d$ panels show the merged and DIC images.

Additional file 2: Figure S2. Negative control without any probe. No signal was detected in the negative control. a and $d$ panels show the merged and DIC images.

Additional file 3: Figure S3. FISH staining of Arsenophonus in whole mount of whitefly B. tabaci at different laser settings. At low laser settings, the signal produced by DNA probe for Arsenophonus was not detectable (A.b). While LNA probe at the same settings could easily detect bacteria, giving good signal and minimum or no background (B.b). But when laser power was increased such that DNA probe signal could be detected, the LNA probe showed very high signal sensitivity and background (C.b). a and c panels show the merged and DIC images.

Additional file 4: Figure S4. FISH staining of Arsenophonus in whole mount of whitefly B. tabaci at low probe concentration. Following the protocol as described, at lower probe concentration (0.6 pmoles) we could not detect Arsenophonus using DNA probe (A.b). LNA probe detects Arsenophonus at the same probe concentration (B.b). a and c panels show the merged and DIC images of the respective probes.

\section{Authors' contributions}

NGP and NP collected the samples. NGP performed the experiments, analyzed the data and wrote the paper. RR edited the paper and designed the research. All authors read and approved the final manuscript.

\section{Acknowledgements}

We are grateful to NAIP, Indian Council for Agricultural Research, Govt. of India for financing this work. NP acknowledges the JRF support received from CSIR, Govt. of India. We would like to thank Mr. Ashok for his valuable laboratory assistance.

Received: 31 October 2011 Accepted: 2 May 2012

Published: 24 May 2012

\section{References}

1. McFadden Gl: Methods Cell Biol 1995, 49:165-183

2. Matsuyama H, Pan Y, Skoog L, Tribukait B, Naito K, Ekman P, Lichter P, Bergerheim US: Deletion mapping of chromosome $8 p$ in prostate cancer by fluorescence in situ hybridization. Oncogene 1994, 9:3071-3076.
3. Huang SF, Xiao S, Renshaw A, Loughlin KR, Hudson TJ, Fletcher J: Fluorescence in situ hybridization evaluation of chromosome deletion patterns in prostate cancer. Am J Pathol 1996, 149(5):1565-1573.

4. Koga R, Tsuchida T, Fukatsu T: Quenching autofluorescence of insect tissues for in situ detection of endosymbionts. Appl Entomol Zool 2009, 44 (2):281-291.

5. Olsen KN, Henriksen M, Bisgaard M, Nielsen OL, Christensen H: Investigation of chicken intestinal bacterial communities by $16 \mathrm{~S}$ rRNA targeted fluorescence in situ hybridization. A Van Leeuw J Microb 2008, 94(3):423-437.

6. West NJ, Schönhuber WA, Fuller NJ, Amann RI, Rippka R, Post AF, Scanlan DJ: Closely related Prochlorococcus genotypes show remarkably different depth distributions in two oceanic regions as revealed by in situ hybridization using $16 \mathrm{~S}$ rRNA-targeted oligonucleotides. Microbiology 2001, 47(Pt 7):1731-1744. Reading, England.

7. Sarma K, Levasseur P, Aristarkhov A, Lee JT: Locked Nucleic Acids (LNAs) reveal sequence requirements and kinetics of Xist RNA localization to the X chromosome. Proc Natl Acad Sci USA 2010, 107(51):22196-22201.

8. Koshkin AA, Nielsen P, Meldgaard M, Rajwanshi VK, Singh SK, Wengel J: LNA (Locked Nucleic Acid): an RNA mimic forming exceedingly stable LNA: LNA duplexes. J Am Chem Soc 1998, 120:13252-13253.

9. Wengel J, Petersen M, Frieden M, Troels K: Chemistry of Locked Nucleic Acids (LNA): design, synthesis, and biophysical properties. Lett Peptide Sci 2003, 10:237-253.

10. Obika S: Synthesis of 2'-O,4'-C-methyleneuridine and -cytidine. Novel bicyclic nucleosides having a fixed C3,-endo sugar puckering. Tetrahedron Lett 1997, 38:8735-8738.

11. Válóczi A, Hornyik C, Varga N, Burgyán J, Kauppinen S, Havelda Z: Sensitive and specific detection of microRNAs by northern blot analysis using LNA-modified oligonucleotide probes. Nucleic Acids Res 2004, 32(22):e175

12. Kubota K, Ohashi A, Imachi H, Harada H: Improved in situ hybridization efficiency with locked-nucleic-acid-incorporated DNA probes. Appl Environ Microbiol 2006, 72(8):5311-5317.

13. Darnell DK, Stanislaw S, Kaur S, Antin PB: Whole mount in situ hybridization detection of mRNAs using short LNA containing DNA oligonucleotide probes. RNA 2010, 16:632-637.

14. Wienholds E, Kloosterman WP, Miska E, Alvarez-Saavedra E, Berezikov E, de Bruijn E, Horvitz HR, Kauppinen S, Plasterk RH: MicroRNA expression in zebrafish embryonic development. Science 2005, 309:310-311.

15. Nelson PT, Baldwin DONA, Kloosterman WP, Kauppinen S, Plasterk RHA, Mourelatos Z: RAKE and LNA-ISH reveal microRNA expression and localization in archival human brain. RNA 2006, 12:187-191.

16. Ason B, Darnell DK, Wittbrodt B, Berezikov E, Kloosterman WP, Wittbrodt J, Antin Parker B, Ronald HA: Plasterk: differences in vertebrate microRNA expression. Proc Natl Acad Sci USA 2006, 103(39):14385-14389.

17. Monotone KT, Feldman MD: In situ detection of Aspergillus ribosomal rRNA sequences using a Locked Nucleic Acid (LNA) probe. Diagn Mol Pathol 2009, 18(4):239-242.

18. Montone KT: Differentiation of Fusarium from Aspergillus species by colorimetric in situ hybridization in formalin-fixed, paraffin-embedded tissue sections using dual fluorogenic-labeled LNA Probes. Am J Clin Pathol 2009, 132(6):866-870.

19. Montone KT, Litzky LA, Feldman MD, Peterman H, Mathis B, Baliff J, Kaiser LR, Kucharczuk J, Nachamkin I: In Situ hybridization for coccidioides immitis 5.8 S ribosomal RNA sequences in formalin-fixed, paraffinembedded pulmonary nodules using a Locked Nucleic Acid (LNA) probe: a rapid means for speciation in tissue sections. Diagn Mol Pathol 2010, 19(2):99-104.

20. Gottlieb Y, Zchori-Fein E, Mozes-Daube N, Kontsedalov S, Skaljac M, Brumin M, Sobol I, Czosnek H, Vavre F, Fleury F, Ghanim M: The transmission efficiency of tomato yellow leaf curl virus by the whitefly Bemisia tabaci is correlated with the presence of a specific symbiotic bacterium species. J Virol 2010, 84:9310-9317.

21. Gottlieb Y, Ghanim Murad, Chiel E, Gerling D, Portnoy V, Steinberg S, Tzuri G, Horowitz AR, Belausov E, Mozes-Daube N, Kontsedalov S, Gershon M, Gal S, Katzir N, Zchori-Fein E: Identification and Localization of a Rickettsia sp. in Bemisia tabaci (Homoptera: Aleyrodidae). Appl Environ Microbiol 2006, 72(5):3646-3652.

22. Gottlieb Y, Ghanim M, Gueguen G, Kontsedalov S, Vavre F, Fleury F, ZchoriFein $E$ : Inherited intracellular ecosystem: symbiotic bacteria share bacteriocytes in whiteflies. FASEB J 2008, 22:2591-2599. 
23. Baumann P: Biology bacteriocyte-associated endosymbionts of plant sapsucking insects. Annu Rev Microbiol 2005, 59:155-189.

24. Ghanim M, Rosell RC, Campbell LR, Czosnek H, Brown JK, Ullman DE: Digestive, salivary, and reproductive organs of Bemisia tabaci (Gennadius) (Hemiptera: Aleyrodidae) B type. J Morphol 2001, 248(1):22-40.

25. Skaljac M, Zanic K, Ban SG, Kontsedalov S, Ghanim Murad: Co-infection and localization of secondary symbionts in two whitefly species. BMC Microbiol 2010, 10:142.

26. Quevedo B, Giertsen E, Zijnge V, Lüthi-Schaller H, Guggenheim B, Thurnheer $T$, Rudolf Gmür: Phylogenetic group- and species-specific oligonucleotide probes for single-cell detection of lactic acid bacteria in oral biofilms. BMC Microbiol 2011, 11:14

27. McTigue PM, Peterson RJ, Kahn JD: Sequence-dependent thermodynamic parameters for locked nucleic acid (LNA)-DNA duplex formation. Biochemistry 2004, 43(18):5388-5405.

28. Thomsen R, Nielsen PS, Jensen TH: Dramatically improved RNA in situ hybridization signals using LNA-modified probes. RNA 2005, 11(11):745-1748.

29. Stoll S, Feldhaar H, Fraunholz MJ, Gross R: Bacteriocyte dynamics during development of a holometabolous insect, the carpenter ant Camponotus floridanus. BMC Microbiol 2010, 10(1):308.

doi:10.1186/1471-2180-12-8

Cite this article as: Priya et al:: LNA probes substantially improve the detection of bacterial endosymbionts in whole mount of insects by fluorescent in-situ hybridization. BMC Microbiology 2012 12:81.

\section{Submit your next manuscript to BioMed Central and take full advantage of:}

- Convenient online submission

- Thorough peer review

- No space constraints or color figure charges

- Immediate publication on acceptance

- Inclusion in PubMed, CAS, Scopus and Google Scholar

- Research which is freely available for redistribution 